\title{
Old and New Players in Oral Antiplatelet Therapy in Treatment of Acute Coronary Syndrome
}

\author{
Kohli $S C^{*}$ \\ ${ }^{1}$ Department of Medicine, Manipal College of Medical sciences, Pokhara, Nepal
}

\section{* Corresponding Author: \\ Prof. Suresh Chandra Kohli \\ Department of Medicine \\ Manipal Teaching Hospital \\ Email: profsckohli@rediffmail.com}

\section{Citation}

Kohli SC. Old and new players in oral antiplatelet therapy in acute coronary syndromes. Nepal Journal of Medical Sciences. 2012; 1(1): 49-55.

\begin{abstract}
Oral antiplatelet therapy plays an important role in treating patients with acute coronary syndrome (ACS), including patients with unstable angina (UA), non-ST segment elevation myocardial infarction (NSTEMI) and patients with ST-segment elevation myocardial infarction (STEMI). All antiplatelet drugs in addition to inhibiting acute arterial thrombosis have danger of interfering with the physiologic role of platelet hemostasis. Bleeding is a major factor in evaluating the utility of available and upcoming antiplatelet drugs and their combination regimes. The role of anti platelet agents in the treatment of ACS has undergone significant changes over the past several years. Aspirin, thienopyridines, and glycoprotein (GP) IIb/IIIa inhibitors are now standard parts of the treatment of STEMI, NSTEMI and UA whether an early invasive or an initial conservative strategy is chosen. Antiplatelet drugs have an important role in secondary prevention in the patients of ischaemic heart disease.
\end{abstract}

Keywords: Acute Coronary Syndrome; antiplatelet therapy; thienopyridines.

\section{Introduction}

As the intricacies of platelet biology and mechanisms of thrombus formation are revealed, novel antiplatelet therapies have emerged. Platelets play a key role in the pathogenesis of atherosclerosis and atherothrombosis, contributing to both the initiation and progression of coronary artery disease. Antiplatelet therapy therefore features prominently in the treatment of acute coronary syndromes (ACS) and in patients undergoing percutaneous coronary intervention. As our understanding of platelet biology continues to evolve, new targets for the inhibition of platelet function have emerged. A computerised medical literature search of all English language articles was done from the "PubMed" online database with the keywords "antiplatelet therapy", "thienopyridines", "acute coronary syndromes" and "proton pump inhibitors".

\section{Irreversible platelet cyclooxygenase-1 inhibitors}

\section{Aspirin}

Aspirin is most widely used antiplatelet agent and serves as the foundation of most strategies. After years of use and numerous clinical trials, the role of aspirin for patients Aspirin with ACS is well established. ${ }^{1}$ It produces its antithrombotic effect by irreversibly acetylating and inhibiting platelet cyclooxygenase (COX)-1, a critical enzyme in the biosynthesis of thromboxane $A_{2}$, a potent platelet activator. ${ }^{1-3}$ Long-term aspirin therapy confers a conclusive net benefit on the risk of subsequent myocardial infarction (MI), stroke, or vascular death among subjects with an intermediate-to-high risk of vascular complications. These include patients with chronic stable angina, patients with prior $\mathrm{MI},{ }^{2}$ patients with unstable angina, ${ }^{1,2}$ as well as 
other high-risk categories. ${ }^{1}$ The proportional effects of longterm aspirin therapy on vascular events in these different clinical settings are rather homogenous, ranging between $20 \%$ and $25 \%$ odds reduction based on an overview of all randomized trials. ${ }^{1}$ Aspirin is also used for primary prevention in patients whose estimated annual risk of MI is $>1 \%$, a point where its benefits are likely to outweigh harms. In men, aspirin mainly reduces the risk of MI, while in women aspirin lowers the risk of stroke. ${ }^{4}$ Aspirin doses of $75-100 \mathrm{mg}$ are recommended for most indications. ${ }^{5}$ However, its low potency due to a partial inhibition of platelet aggregation, some patients resistance to aspirin, and its gastrointestinal side effects prompted the search for more consistent, and more specific platelet inhibiting agents..$^{5-7}$

\section{Irreversible P2Y12 purinoreceptors inhibitors}

\section{Clopidogrel}

The key role of adenosine diphosphate (ADP) in the process of platelet aggregation, and thrombus formation led to the development of drugs targeting P2Y12 receptor. ${ }^{8}$ The thienopyridine class of antiplatelets-Ticlopidine, Clopidogrel, and Prasugrel- selectively and irreversibly inhibit the P2Y12 purinoreceptors throughout the lifespan of the platelet. Clopidogrel is an irreversible thienopyridine ADP receptor antagonist. It can be considered an alternative to aspirin in patients with aspirin intolerance or who are allergic to aspirin. ${ }^{9}$ In patients with established atherosclerotic cardiovascular $(\mathrm{CV})$ disease. Clopidogrel alone reduced (5.8\% to $5.3 \%)$, the combined risk of major $\mathrm{CV}$ events, ischemic stroke, MI, and vascular death compared with aspirin alone $^{6}$ and led to less (GI) bleeding (2.7\% to $2.0 \%$ ). Clopidogrel is recommended as an alternative agent for patients with $\mathrm{CV}$ disease unable to take aspirin. ${ }^{9-11}$

Clopidogrel and Aspirin are combined to capitalize on their capacity to block complementary pathways on platelet activation. ${ }^{10-13}$ The combination of Clopidogrel and Aspirin is also effective in patients with unstable angina. ${ }^{4}$ Thus in such patients, the risk of cardiovascular death, MI or stroke was $9.3 \%$ in those randomized to combination of Clopidogrel and Aspirin and $11.4 \%$ in those given aspirin alone. This relative risk reduction with combination was highly statistically significant. ${ }^{4,14,15}$ In primary prevention settings, dual antiplatelet therapy with Clopidogrel and aspirin did not significantly reduce major $\mathrm{CV}$ events compared to aspirin alone $(6.8 \%)$ versus $(7.3 \%)$ but increased severe bleeding $(1.3 \%$ to $1.7 \%)$. Therefore, the combination of Clopidogrel and aspirin should only be used when there is a clear benefit. ${ }^{13,14}$

All the orally administered thienopyridines are prodrugs which require biotransformation into their active metabolites by fixed sets of hepatic cytochrome P450 (CYP) enzymes, in particular, the CYP3A4 isozyme. The limitations of Clopidogrel - delayed onset of action, large interindividual variability in platelet response, irreversibility of its inhibitory effect on platelet, the two-step activation process, involving a series of cytochrome P-450 (CYP) isoenzymes, rendering it susceptible to the interference of genetic polymorphisms ${ }^{16-}$ ${ }^{18}$ and drug-drug interactions ${ }^{17}$ encouraged the investigation of novel antiplatelet agents. ${ }^{19,20}$

\section{Prasugrel}

Prasugrel is a new thienopyridine derivative with a rapid onset and consistent inhibition of platelet aggregation. In contrast to Clopidogrel's esterase inactivation and two-step CYP-dependent activation, Prasugrel has a more efficient and simpler metabolism, which requires only one reaction by the liver enzymes to yield its active metabolite..$^{21-23}$ This difference in the metabolism of these thienopyridines translates clinically into different patient responses and drug interactions with these antiplatelet agents. ${ }^{17}$ Patients with a poor response to Clopidogrel have an increased risk of coronary thrombosis. ${ }^{19}$ The increased risk of bleeding due to prolonged persistence of the Clopidogrel effect is of concern when patients need non-deferrable surgery such as urgent coronary-artery bypass grafting (CABG). To compare Prasugrel with Clopidogrel, in TRITON-TIMI 38 trial, ${ }^{23} 13,608$ patients were randomly assigned with moderate-to-high-risk acute coronary syndromes with scheduled percutaneous coronary intervention (PCI) to receive Prasugrel (a 60-mg loading dose and a 10-mg daily maintenance dose) or Clopidogrel (a 300-mg loading dose and a 75-mg daily maintenance dose) for 6 to 15 months. The primary efficacy end point was death from cardiovascular causes, nonfatal myocardial infarction or nonfatal stroke. The key safety end point was major bleeding. In patients with ACS and planned PCI, Prasugrel reduced major CV events from $12.1 \%$ to $9.9 \%$ compared with Clopidogrel but increased major bleeding from $1.8 \%$ to $2.4 \%$ and fatal bleeding from $0.1 \%$ to $0.4 \%$. Because patients older than age 75 years and those with a history of prior stroke or transient ischemic attack have a particularly high risk of bleeding, Prasugrel should generally be avoided in older patients, and the drug is contraindicated in those with a history of cerebrovascular disease. Caution is required if Prasugrel is used in patients weighing less than $60 \mathrm{~kg}$ or in those with renal impairment.

\section{Reversible P2Y12 receptor inhibitor}

\section{Ticagrelor}

However, the limitations of the irreversibility of the thienopyridine effect are also evident with Prasugrel. Ticagrelor, is a novel, reversible, direct-acting P2Y12 receptor blocker. Report on the results of the study of 
Platelet Inhibition and Patient outcome - $\mathrm{PLATO}^{24}$ showed the reduced primary endpoint of vascular death, MI, or stroke from $11.7 \%$ to $9.8 \%$ compared with Clopidogrel, with no significant difference in major bleeding $(11.6 \%$ versus $11.2 \%$ ) but with an increased risk of noncoronary artery bypass graft major bleeding (3.8\% to 4.5\%). PLATO ${ }^{24}$ is the third randomized trial evaluating novel antagonist of the platelet ADP receptors in patients with acute coronary syndromes, following the Clopidogrel in Unstable Angina to Prevent Recurrent Events - CURE trial ${ }^{9}$ and TRITONTIMI 38. ${ }^{23}$ Two striking differences among the outcomes of these three trials deserve special consideration. First, in both the CURE trial and TRINTON-TIMI 38, ${ }^{23}$ stronger platelet inhibition was associated with an increased risk of bleeding, whereas in $\mathrm{PLATO}^{24}$ the risk of major bleeding was not increased with Ticagrelor. As compared to Clopidogrel, Ticagrelor was associated with more frequent non-CABG related bleeding but it was safer than Clopidogrel in patients undergoing CABG. Genetic variations in CYP isoenzymes do not appear to affect metabolism of Ticagrelor.

In PLATO trial, ${ }^{24}$ Ticagrelor was associated with a higher rate of major bleeding not related to coronary-artery bypass grafting ( $4.5 \%$ vs. $3.8 \%, \mathrm{P}=0.03)$, including more instances of fatal intracranial bleeding and fewer of fatal bleeding of other types. Therefore in patients who have an acute coronary syndrome with or without ST-segment elevation, treatment with ticagrelor as compared with Clopidogrel significantly reduced the rate of death from vascular causes, myocardial infarction, or stroke without an increase in the rate of overall major bleeding but with an increase in the rate of non-procedure-related bleeding. Ticagrelor therapy may also be preferred in patients whose coronary anatomy is unknown and for whom CABG procedure is deemed probable. Dyspnoea occurred more frequently with Ticagrelor than Clopidogrel. Discontinuation of Ticagrelor due to dyspnoea occurred in $0.9 \%$ of patients in Ticagrelor group. Holter monitoring detected more ventricular pauses during the first week in Ticagrelor group than in the Clopidogrel group but these episodes were infrequent at 30 days and were rarely associated with symptoms. There were no significant differences in rates of clinical manifestations of bradyarrhythmias between the two groups. Ticagrelor therapy should be discouraged in patients who have chronic pulmonary disease, hyperuricemia, moderate to severe renal failure, bradyarrhythmias unprotected by pacemaker and history of syncope. ${ }^{24}$ Ticagrelor has been included in 2011 AHA/ACC recommendations for use in ACS. ${ }^{25}$ The availability of three agents for antagonizing platelet ADP receptors may make it possible to individualize antiplatelet therapy. In particular, Ticagrelor therapy may be preferred in patients whose coronary anatomy is unknown and for whom a CABG procedure is deemed probable. ${ }^{26}$

\section{Proton pump inhibitors and antiplatelet agents}

Proton pump inhibitors (PPIs) are often prescribed prophylactically when Clopidogrel is started, to prevent gastrointestinal complications such as ulceration and related bleeding $^{27}$ due to dual-antiplatelet therapy, in particular Acetyl Salicylic Acid (ASA) and Clopidogrel. ${ }^{28}$ PPI medications have been found to interfere with the metabolism of Clopidogrel. ${ }^{29}$ There have been retrospective reports of adverse cardiovascular outcomes (e.g., readmission for ACS) when the antiplatelet regimen of Clopidogrel and ASA is accompanied by PPIs, assessed as a group, compared with the use of this regimen without a PPI. ${ }^{29-31}$ In a retrospective cohort study from the Veterans Affairs' medical records and pharmacy database, concomitant Clopidogrel and PPI therapy (with Omeprazole, Rabeprazole, Lansoprazole, or Pantoprazole) at any time point during follow-up of 8205 patients discharged for ACS was associated with an increased risk of death or rehospitalization for ACS. ${ }^{29}$ Other post hoc study, ${ }^{31}$ a retrospective data analysis from the NHLBI Dynamic Registry ${ }^{32}$ in which PPIs were assessed as a class in combination with a Clopidogrel and an ASA regimen have not found an effect of PPI therapy on the clinical effect of Clopidogrel in ACS patients, after ACS, or in a general post-PCI population respectively.

Some studies have suggested that adverse cardiovascular outcomes with combination of Clopidogrel and a Proton Pump Inhibitor (PPI) are explained the individual PPI, in particular the use of a PPI that inhibits CYP450 2C19, which includes Omeprazole, Lansoprazole and Rabeprazole. The PPI Omeprazole notably has been reported to significantly decrease the inhibitory effect of Clopidogrel on platelet aggregation. ${ }^{33,34}$ One study reported that the PPI Pantoprazole was not associated with recurrent $\mathrm{MI}$, possibly because of lack of inhibition of CYP450 2 C19. ${ }^{30}$

An open-label drug study evaluated the effects of the PPI Lansoprazole on the pharmacokinetics and pharmacodynamics of Prasugrel and Clopidogrel in healthy subjects given single doses of Prasugrel $(60 \mathrm{mg}$ ) and Clopidogrel $(300 \mathrm{mg}$ ) with and without concurrent Lansoprazole $(30 \mathrm{mg})$ per day. The data suggest that inhibition of platelet aggregation was reduced in patients who took the combination of Clopidogrel and Lansoprazole, whereas it was unaffected after a Prasugrel dose. ${ }^{35}$ The PRINCIPLE (Prasugrel In Comparison to Clopidogrel for Inhibition of Platelet Activation and Aggregation) TIMI-44 trial ${ }^{36,37}$ and the TRITON-TIMI 38 trial. The findings indicated that first PPI treatment attenuated the pharmacodynamics effects of Clopidogrel and to a lesser extent those of Prasugrel. Secondly, PPI treatment did not affect the clinical outcome of patients given Clopidogrel or Prasugrel. This finding was true for all PPIs that were studied, including Omeprazole 
and Pantoprazole.

A selected review of the published literature done in a study on the indications for gastric protection with PPI in patients on mono- or dual antiplatelet therapy focusing on the possible interaction between Clopidogrel and PPI revealed that in ex vivo analyses of platelet function, a reduced efficacy of Clopidogrel was observed in patients on comedication with Omeprazole. This was not the case with the comedication of other PPIs. It further stated that "To date, clear evidence is missing to state that comedication with PPI reduces the efficacy of Clopidogrel IN VIVO. If both Clopidogrel and PPI need to be prescribed, a split dosage regimen of PPI in the morning and Clopidogrel in the evening can be recommended. The short half-life of both medications explains the rationale of this recommendation. ${ }^{38}$

The use of $\mathrm{H} 2$ antagonists as an alternative to PPIs in the setting of dual-antiplatelet therapy, although they cannot be relied on to protect as well as PPIs, and there are few data about their use with ASA needs further consideration. The FAMOUS (Famotidine for the Prevention of Peptic Ulcers in Users of Low-Dose Aspirin), a phase II, double-blind, randomized, placebo-controlled trial, found that among patients with a history of coronary heart disease, diabetes mellitus, or cerebrovascular disease who were taking lowdose ASA, 12 weeks of Famotidine $20 \mathrm{mg}$ twice daily $(n=204)$ compared with placebo twice daily $(n=200)$ was beneficial in reducing the incidence of peptic ulcer or oesophagitis during follow-up endoscopy at 12 weeks. In the Famotidine group, Clopidogrel use was 19\% and Dipyridamole use was $6 \%{ }^{39}$

AHA / ACC focused update 2009 has noted that additional data, notably randomized controlled clinical trial data that have been peer reviewed and published, are needed before an official recommendation can be made about the use of dual antiplatelet therapy with PPIs in the setting of ACS. ${ }^{40}$

European College of Cardiology (ESC) has issued the following recommendations for oral antiplatelet agents in the management of acute coronary syndromes in $2011 .{ }^{41}$

\section{Esc Recommendations for Oral Antiplatelet Agents}

Aspirin should be given to all patients without contraindications at an initial loading dose of 150-300 mg, and at a maintenance dose of 75-100 mg daily long-term regardless of treatment strategy.

\section{Class I}

- A P2Y12 inhibitor should be added to aspirin as soon as possible and maintained over 12 months, unless there are contraindications such as excessive risk of bleeding. ${ }^{42,43}$
- A proton pump inhibitor (preferably not Omeprazole) in combination with DAPT is recommended in patients with a history of gastrointestinal haemorrhage or peptic ulcer, and appropriate for patients with multiple other risk factors (Helicobacter pylori infection, age $\geq 65$ years, concurrent use of anticoagulants or steroids)..$^{9,23,24}$

- $\quad$ Prolonged or permanent withdrawal of P2Y12 inhibitors within 12 months after the index event is discouraged unless clinically indicated. ${ }^{28,44,45}$

- Prasugrel (60-mg loading dose, 10-mg daily dose) is recommended for P2Y12-inhibitor-naïve patients (especially diabetics) in whom coronary anatomy is known and who are proceeding to PCI unless there is a high risk of life threatening bleeding or other contraindications. $^{24}$

- Clopidogrel (300-mg loading dose, 75-mg daily dose) is recommended for patients who cannot receive Ticagrelor or Prasugrel. ${ }^{23}$

- A 600-mg loading dose of Clopidogrel (or a supplementary 300-mg dose at PCI following an initial 300-mg loading dose) is recommended for patients scheduled for an invasive strategy when Ticagrelor or Prasugrel is not an option. ${ }^{9,46,47}$

- A higher maintenance dose of Clopidogrel $150 \mathrm{mg}$ daily should be considered for the first 7 days in patients managed with PCI and without increased risk of bleeding. ${ }^{19,43,48}$

\section{Class IIa}

- Increasing the maintenance dose of Clopidogrel based on platelet function testing is not advised as routine, but may be considered in selected cases. ${ }^{44}$

\section{Class IIb}

- Genotyping and/or platelet function testing may be considered in selected cases when Clopidogrel is used. ${ }^{49}$

\section{Class IIb}

- In patients pre-treated with P2Y12 inhibitors who need to undergo non-emergent major surgery (including $\mathrm{CABG}$ ), postponing surgery at least for 5 days after cessation of Ticagrelor or Clopidogrel, and 7 days for Prasugrel, if clinically feasible and unless the patient is at high risk of ischaemic events should be considered. ${ }^{50,51}$

Abbreviations: CABG: coronary artery bypass graft; COX: cyclo-oxygenase; DAPT -dual (oral) antiplatelet therapy; NSAID -non-steroidal anti-inflammatory drug; PCI: percutaneous coronary intervention. 


\section{References:}

1. Baigent C, Sudlow C, Collins R, et al. Collaborative meta-analysis of randomized trials of antiplatelet therapy for prevention of death, myocardial infarction, and stroke in high-risk patients. BMJ 2002;324:71-86.

2. Mukherjee D, Nissen SE, Topol EJ, et al. Risk of cardiovascular events associated with selective COX2 inhibitors. JAMA 2001;286:954-9.

3. Baigent C, Patrono C. Selective cyclooxygenase-2 inhibitors, aspirin and cardiovascular disease: a reappraisal. Arthritis Rheum 2003;48:12-20.

4. Weitz JI. Antiplatelet, Anticoagulant, and Fibrinolytic Drugs. In: Longo MD, Kasper DL, Loscalzo J et al. Harrison's Principles of Internal Medicine. Volume-1. 18th ed. New York: Mc Graw-Hills, 2011:988-1004.

5. Jennings LK. Role of platelets in atherothrombosis. Am J Cardiol 2009;103:4-10.

6. Awtry EA, Loscalzo J. Cardiovascular drugs - aspirin. Circulation 2000;101:1206-18.

7. Patrono C, Coller B, FitzGerald GA, et al. Plateletactive drugs: the relationships among dose, effectiveness, and side effects. The Seventh ACCP Conference on Antithrombotic and Thrombolytic. Chest 2004;126:234-64.

8. Shankar H, Kunapuli SP. Is the P2Y1 receptor a better target for antithrombotic drugs? Drug Discov Today 2005;2:285-90.

9. Yusuf S, Zhao F, Tognoni G, et al. Effects of clopidogrel in addition to aspirin in patients with acute coronary syndromes without ST-segment elevation. N Engl J Med 2001;345:494-502.

10. Chen ZM, Jiang LX, Chen YP, et al. Addition of clopidogrel to aspirin in 45,852 patients with acute myocardial infarction: randomized placebo-controlled trial. Lancet 2005;366:1607-21.

11. Sabatine MS, Cannon CP, Gibson CM, et al. Addition of clopidogrel to aspirin and fibrinolytic therapy for myocardial infarction with ST-segment elevation. N Engl J Med 2005;352:1179-89.

12. Bowry AD, Brookhart MA, Choudhry NK, et al. Meta-analysis of the efficacy and safety of clopidogrel plus aspirin as compared to antiplatelet monotherapy for the prevention of vascular events. Am J Cardiol 2008;101:960-6.

13. Squizzato A, Keller T, Romualdi E, et al. Clopidogrel plus aspirin versus aspirin alone for preventing cardiovascular disease. Cochrane Database of Systematic Reviews 2011, Issue 1.

14. Yusuf S, Zhao F, Mehta SR, et al. Effects of clopidogrel in addition to aspirin in patients with acute coronary syndromes without ST-segment elevation. N Engl J Med 2001;345:494-502.

15. Bhatt DL, Fox KA, Hacke W, et al. Clopidogrel and aspirin versus aspirin alone for the prevention of atherothrombotic events. $\mathrm{N}$ Engl $\mathrm{J}$ Med 2006;354:1706-17.

16. Mega JL, Close SL, Wiviott SD, et al. Cytochrome P-450 polymorphisms and response to clopidogrel. N Engl J Med 2009;360:354-62.

17. Gilard M, Arnaud B, Cornily JC, et al. Influence of omeprazole on the antiplatelet action of clopidogrel associated with aspirin: the randomized, double-blind OCLA (Omeprazole CLopidogrel Aspirin) study. J Am Coll Cardiol 2008;51:256-60.

18. Brandt JT, Close SL, Iturria SJ, et al. Common polymorphisms of CYP2C19 and CYP2C9 affect the pharmacokinetic and pharmacodynamic response to clopidogrel. Eur Heart J. 2009;30:1744-52.

19. Snoep JD, Hovens MM, Eikenboom JC, et al. Clopidogrel nonresponsiveness in patients undergoing percutaneous coronary intervention with stenting: a systematic review and meta-analysis. Am Heart J 2007;154:221-31.

20. Gori AM, Marcucci R, Migliorini A, et al. Incidence and clinical impact of dual nonresponsiveness to spirin and clopidogrel in patients withdrug-eluting stents. J Am Coll Cardiol. 2008;52:734-9.

21. Wallentin L, Varenhorst C, James S, et al. Prasugrel achieves greater and faster $\mathrm{P} 2 \mathrm{Y} 12$ receptor mediated platelet inhibition than clopidogrel due to more efficient generation of its active metabolite in aspirintreated patients with coronary artery disease. Eur Heart J. 2008;29:21-30.

22. Brandt J T, Close SL, Iturria SJ, et al. Common polymorphisms of CYP2C19 and CYP2C9 affect the pharmacokinetic and pharmacodynamic response to clopidogrel but not prasugrel. J Thromb Haemost 2007;5:2429-36.

23. Wiviott SD, Braunwald E, McCabe CH, et al. Prasugrel versus clopidogrel in patients with acute coronary syndromes. N Engl J Med 2007;357:2001-15. 
24. Wallentin L, Becker RC, Budaj A, et al. Ticagrelor versus clopidogrel in patients with acute coronary syndromes N Engl J Med 2009;361:1045-57.

25. Levine GN, Bates ER, Blankenship JC, et al. 2011 ACCF/AHA/SCAI guideline for percutaneous coronary intervention: a report of the American College of Cardiology Foundation/American Heart Association Task Force on Practice Guidelines and the Society for Cardiovascular Angiography and Interventions. Circulation 2011: published online before print November 7, 2011, 10.1161/CIR.0b013e31823ba622.

26. Albert Schomig Ticagrelor- Is there need for a new player in the antiplatelet-therapy field ? N Engl J Med 2009;361:1108-11.

27. Abraham NS, Hlatky MA, Antman EM, et al. ACCF/ ACG/AHA 2010 Expert Consensus Document on the concomitant use of proton pump inhibitors and thienopyridines: a focused update of the ACCF/ ACG/AHA 2008 expert consensus document on reducing the gastrointestinal risks of antiplatelet therapy and NSAID use: a report of the American College of Cardiology Foundation Task Force on Expert Consensus Documents. Circulation 2010;122:2619-33.

28. Donoghue ML, Braunwald E, Antman EM, et al. Pharmacodynamic effect and clinical efficacy of clopidogrel and prasugrel with or without a protonpump inhibitor. Lancet 2009;374: 989-97.

29. Ho PM, Maddox TM, Wang L, et al. Risk of adverse outcomes associated with concomitant use of clopidogrel and proton pump inhibitors following acute coronary syndrome. JAMA 2009;301: 937-44.

30. Juurlink DN, Gomes T, Ko DT, et al. A populationbased study of the drug interaction between proton pump inhibitors and clopidogrel. CMAJ 2009;180: 713-8.

31. Simon T, Verstuyft C, Mary-Krause $M$, et al. Genetic determinants of response to clopidogrel and cardiovascular events. N Engl J Med 2009;360: 36375.

32. Ramirez JF, Selzer F, Chakaprani R. Proton pump inhibitor and clopidogrel combination is not associated with adverse clinical outcomes after PCI: the NHLBI Dynamic Registry. J Am Coll Cardiol 2009;53: 27-8.

33. Gilard M, Arnaud B, Cornily JC, et al. Influence of omeprazole on the antiplatelet action of clopidogrel associated with aspirin: the randomized, double-blind
OCLA (Omeprazole, Clopidogrel, Aspirin) study. J Am Coll Cardiol 2008;51:256-60.

34. Sibbing D, Morath $\mathrm{T}$, Stegherr J, et al. Impact of proton pump inhibitors on the antiplatelet effects of clopidogrel. Thromb Haemost 2009;101:714-9.

35. Small DS, Farid NA, Payne CD, et al. Effects of the proton pump inhibitor lansoprazole on the pharmacokinetics and pharmacodynamics of prasugrel and clopidogrel J Clin Pharmacol 2008;48:475-84.

36. Wiviott SD, Trenk D, Frelinger AL, et al. Prasugrel compared with high loading- and maintenance-dose clopidogrel in patients with planned percutaneous coronary intervention: the Prasugrel in Comparison to Clopidogrel for Inhibition of Platelet Activation and Aggregation-Thrombolysis in Myocardial Infarction 44 trial. Circulation 2007;116:2923-32.

37. Aubert RE, Epstein RS, Teagarden JR, et al. Proton pump inhibitors effect on clopidogrel effectiveness: the Clopidogrel Medco Outcomes Study. Circulation 2008;118:S-815.

38. Venerito $M$, Kandulski A, Malfertheiner $P$, et al. Dilemma between gastroprotection and cardiovascular prevention. Dtsch Med Wochenschr 2010;135:2193-8.

39. Taha AS, McCloskey C, Prasad R, et al. Famotidine for the prevention of peptic ulcers and oesophagitis in patients taking low-dose aspirin (FAMOUS). Lancet 2009;374:119-25.

40. Harlan M, Krumholz, Douglass A, et al. ACC/AHA Guidelines for the Management of Patients With STElevation Myocardial Infarction (Updating the 2004 Guideline and 2007 Focused Update) and ACC/ AHA/SCAI Guidelines on Percutaneous Coronary Intervention (Updating the 2005 Guideline and 2007 Focused Update) STEMI and PCI AHA/ACC Focused Updates Circulation. 2009;120:2271-306.

41. Bassand JP, Agewall S, Christian W, et al. ESC Guidelines for the management of acute coronary syndromes in patients presenting without persistent ST-segment elevation. Eur Heart J 2011. First published online: August 26, 2011.

42. Baigent C, Blackwell L, Collins R, et al. Aspirin in the primary and secondary prevention of vascular disease: collaborative meta-analysis of individual participant data from randomised trials. Lancet 2009;373:1849-60.

43. Mehta SR, Tanguay JF, Eikelboom JW, et al. Doubledose versus standard-dose clopidogrel and high-dose versus low-dose aspirin in individuals undergoing 
percutaneous coronary intervention for acute coronary syndromes (CURRENT-OASIS 7). Lancet 2010;376:1233-43.

44. Abraham NS, Hlatky MA, Antman EM, et al. ACCF/ ACG/AHA 2010 Expert Consensus Document on the concomitant use of proton pump inhibitors and thienopyridines: a focused update of the ACCF/ACG/ AHA 2008 expert consensus document on reducing the gastrointestinal risks of antiplatelet therapy and NSAID use. Circulation 2010;122:2619-33.

45. Bhatt DL, Cryer BL, Contant CF, et al. Clopidogrel with or without omeprazole in coronary artery disease. N Engl J Med 2010;363:1909-17.

46. Mehta SR, Yusuf S, Peters RJ, et al. Effects of pretreatment with clopidogrel and aspirin followed by long-term therapy in patients undergoing percutaneous coronary intervention: the PCI-CURE study. Lancet 2001;358:527-33.

47. Steinhubl SR, Berger PB, Mann JT et al. Early and sustained dual oral antiplatelet therapy following percutaneous coronary intervention. JAMA 2002;288:2411-20.
48. Montalescot G, Sideris G, Meuleman C, et al. A randomized comparison of high clopidogrel loading doses in patients with non-ST-segment elevation acute coronary syndromes: the ALBION (Assessment of the Best Loading Dose of Clopidogrel to Blunt Platelet Activation, Inflammation and Ongoing Necrosis) Trial. J Am Coll Cardiol 2006;48:931-8.

49. Price MJ, Berger PB, Teirstein PS, et al. Standardvs high-dose clopidogrel based on platelet function testing after percutaneous coronary intervention: the GRAVITAS randomized trial. JAMA 2011;305:1097-105.

50. Mega JL, Close SL, Wiviott SD, et al. Genetic variants in ABCB1 and CYP2C19 and cardiovascular outcomes after treatment with clopidogrel and prasugrel in the TRITON-TIMI 38 trial: a pharmacogenetic analysis. Lancet 2010;376:1312-9.

51. Breet N, Werkum J, Bouman H, et al. Comparison of platelet function tests in predicting clinical outcome in patients undergoing coronary stent implantation. JAMA 2010;303:754-62. 